\title{
An Improved Event Plane Detector for the STAR Experiment
}

\author{
Prashanth Shanmuganathan* \\ (for the STAR Collaboration) \\ Lehigh University, USA \\ E-mail: prs416@lehigh.edu
}

\begin{abstract}
These proceedings present the design, construction and engineering run status of the Event Plane Detector (EPD) upgrade at the STAR experiment. The EPD is a key detector for the upcoming beam energy scan (BES-II) program at the STAR experiment. During the first phase of the beam energy scan (BES-I), the STAR collaboration investigated observables as a of function beam energy in order to map the quantum chromodynamics (QCD) phase diagram. Some of these observables showed hints of non-monotonic behavior near the expected critical point location and first-order phase transition. But limited statistics, acceptance, and poor event plane resolution did not allow firm conclusions for a discovery. Therefore, BES-II has been approved, and STAR will take data in 2019 and 2020, with several detector and accelerator upgrades. The EPD is one such upgrade to the existing detector, which replaces the existing Beam-Beam Counter detector with larger granularity and acceptance. It can provide collision centrality measurements independent of the main tracking detectors. The EPD is capable of providing better event plane resolution and allows STAR to trigger on high luminosity collisions at lower energies, so that significant enhancements of measurements can be achieved.
\end{abstract}

Critical Point and Onset of Deconfinement - CPOD2017

7-11 August, 2017

The Wang Center, Stony Brook University, Stony Brook, NY

${ }^{*}$ Speaker. 


\section{Motivation for an Event Plane Detector}

The Beam Energy Scan (BES) program at the Relativistic Heavy-Ion Collider has surveyed the QCD phase diagram over the range $\sqrt{s_{N N}}=7.7$ to $200 \mathrm{GeV}$. At the top RHIC energy, there is evidence of a crossover type phase transition from quark matter to hadronic matter [1]. While at lower beam energies, typically $\sqrt{s_{N N}}=20 \mathrm{GeV}$, additional features of the QCD phase diagram, like a possible first-order phase transition and a critical point, may come into play $[1,2]$. The product of $4^{\text {th }}$ and $2^{\text {nd }}$ moments of the net proton distributions showed an energy dependent non-monotonic behavior near the predicted critical point [3]. However the observed behavior is extremely sensitive to the phase-space studied, a small change in phase-space showed variations in results [4]. The main drawback was using part of the acceptance of the TPC for the collision centrality estimation, and therefore limited the phase-space used in the analysis. Earlier hydrodynamic calculations [9, 10] suggested the minimum in baryon directed flow slope $d v_{1} / d y$ versus beam energy as a signature of a first-order phase transition between hadronic matter and quark-gluon plasma (QGP). STAR's measurements for proton and $\Lambda$ baryons $d v_{1} / d y$ shows a minimum at lower RHIC energies [5, 6]. Global polarization of $\Lambda$ hyperon used as a probe to measure vorticity of QGP created in a heavyion collision and recent measurements from STAR showed positive polarization for $\Lambda$ and $\bar{\Lambda}$ [7]. Poor event plane resolution and large statistical uncertainty prevent strong conclusions to be made from these measurements.

Considering the importance of a systematic scan of the QCD phase diagram, phase two of the beam energy scan was approved to run in 2019 and 2020. Both detectors and the accelerator are being upgraded to improve the measurements, so that quantitative measurements can be made for a discovery. Three main detector upgrades are ongoing at STAR, namely the inner TPC upgrade, the end-cap time-of-flight detector and the event plane detector.

The EPD is proposed to improve the performance of the existing detector beam-beam counters (BBC) [8]. The BBC consist of two arrays of scintillator wheels surrounding the beam pipe, mounted just outside the east and west pole tips of the STAR magnet, $3.75 \mathrm{~m}$ away from the center of the TPC. Figure 1 (a) shows one such wheel. Each BBC detector contains two inner rings and two outer rings of hexagonal scintillator tiles. The two inner rings, which cover pseudorapidity $3.3<|\eta|<5.2$, are the primary tool for most analyses. The BBC is a relatively fast detector, and therefore it is used to trigger events, especially at lower beam energies, and in $p+p$ collisions where there are no spectators. The timing difference between the two BBC modules helps to define the location of the collision vertex along the beam pipe $(z)$. The BBC offers the best method for determining the first-order event plane azimuth for beam energies at and below $39 \mathrm{GeV}$.

EPD has very similar functionality as the BBC. But, its higher granularity and acceptance offers improved performance of the detector (see panel (b) of Fig. 1). For example, increased radial segmentation allows the collision centrality to be calculated in the forward region. This allows use of the full rapidity range of the main tracking detector and eliminates possible auto correlations in analyses. Centrality estimation is not possible with the inner tiles of the BBC. Another example is the higher azimuthal segmentation, it improves the first order event plane resolution by at least a factor of two. Initial simulations studies showed the higher order event plane can also be reconstructed with reasonable resolution. In addition, the higher granularity brings a new frontier to STAR's measurements, for example $d N / d \eta$ measurements at forward rapidity. The EPD is an 
important detector for STAR's fixed-target program, as it provides full $\eta$ coverage in the projectile rapidity.

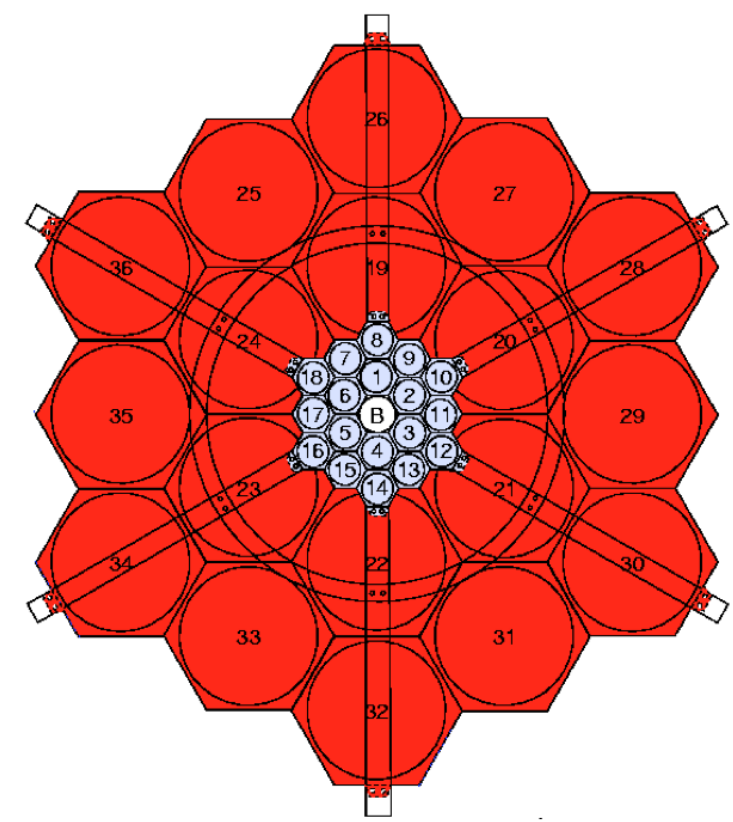

(a) Beam-Beam Counters

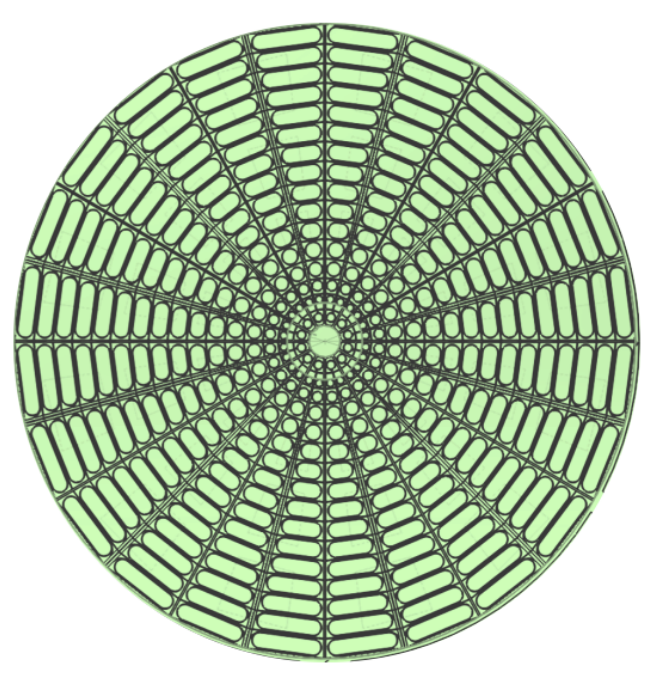

(b) Event Plane Detector

Figure 1: On the left is a schematic of single wheel of the beam-beam counter (BBC). On the right is a schematic view of a single EPD wheel. Both the BBC and the EPD wheels are sketched to match the scale.

\section{Design and Construction}

Two EPD wheels are to be placed in the location of the BBC wheels $(z= \pm 3.75 \mathrm{~m}$ from the center of TPC). Radially the wheels extend from $4.5 \mathrm{~cm}$ to $90 \mathrm{~cm}$ from the beam axis, which cover pseudorapidity $2.1<|\eta|<5$.1. An EPD wheel has 16 radial segments. The radial segmentation is driven by many aspects like directed flow, $z$-vertex position, trigger and so on. The innermost radial segment has 12 azimuthal divisions spanning an angle of $30^{\circ}$. The rest of the segments have 24 azimuthal divisions spanning an angle of $15^{\circ}$. The azimuthal segmentation is driven by higher flow harmonics. The segmentation is optimized for less than $10 \%$ multi-hit probability in the same tile at $19.6 \mathrm{GeV}$ using PHOBOS and UrQMD $d N / d y$ measurements [11]. Monte Carlo calculations and other details to optimize the segmentation are explained in reference [12]. The EPD has 372 tiles (372 channels) in a wheel, while the BBC has only 36 tiles ( 24 channels) in a wheel. Figure 1 illustrates the BBC and EPD tile geometry.

A EPD wheel is composed of 12 "super-sectors". A super-sector is a single $1.2 \mathrm{~cm}$ thick Eljen scintillator wedge (Eljen EJ-200), milled and optically separated to form 31 tiles, as follows. To create isolated individual tiles, first isolation grooves are milled on the back side of a super-sector scintillator wedge halfway through (1.65 wide and $6 \mathrm{~mm}$ deep) according to the tile structure shown in Fig. 2 panel (a). The isolation grooves are epoxied with $\mathrm{TiO}_{2}$ doped DER-332 epoxy to keep 


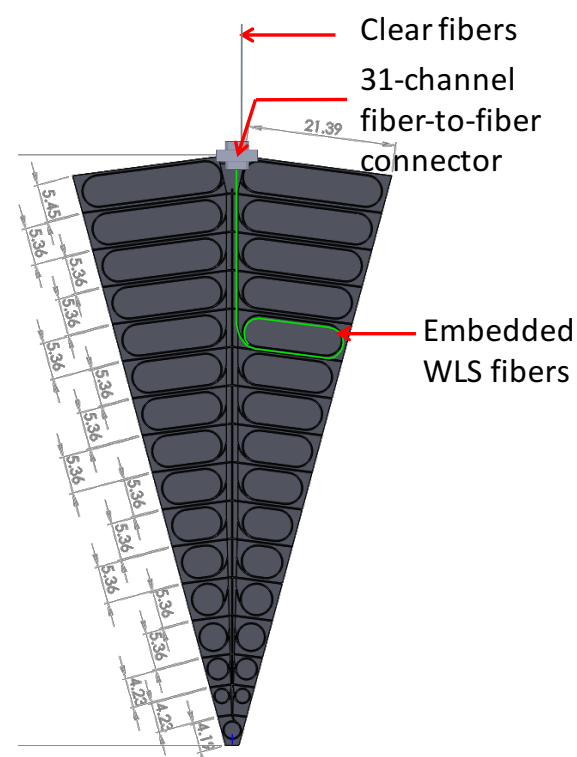

(a)

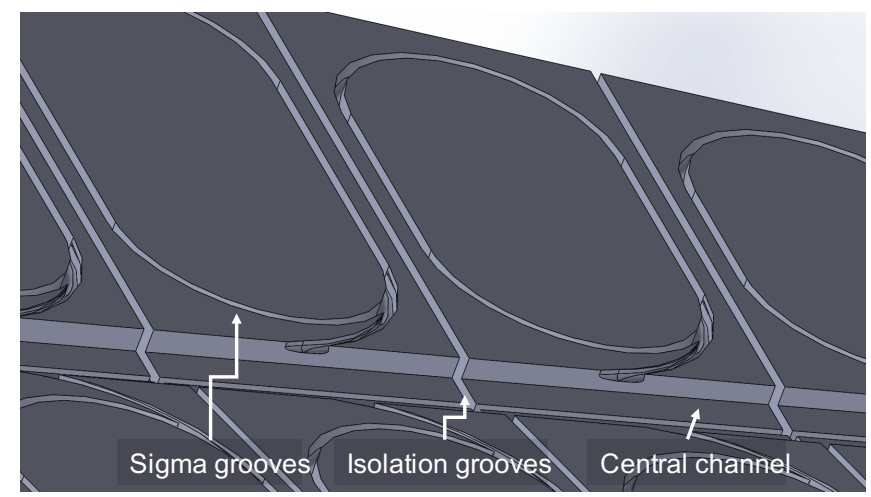

(b)

Figure 2: Left panel shows computer aid drawing of single super-sector, the path of wavelength shifting fiber in a tile and location of edge connector. The dimensions of tiles are in inches. The right panel is a computer aid drawing shows the isolation grooves, sigma grooves and milled out central channel in a super sector.

them in place and to provide light insulation from neighboring tiles [13] . Then the front side is milled for the remaining half of the isolation groves, sigma shaped grooves for the wavelength shifting fibers, and a central one to route the wavelength shifting fibers to the edge of the supersector. The milled front side of the super-sector is shown in Fig, 2 panel (b). Wavelength shifting fibers are used to collect light from the EPD tiles, and to transport the signal to the edge of the supersector. Three turns of wavelength shifting fibers (Kuraray Y-11, $1.0 \mathrm{~mm}$ in diameter) are embedded in the sigma grooves, routed to the edge of super-sector. Then the wavelength shifting fibers are glued with optical cement epoxy (Eljen EJ-500) to place in sigma groove. Parts of the wavelength shifting fibers outside the sigma groove are painted with reflective paint (Eljen: EJ-510), so the fibers will collect light only from its associated tile. Also the ends of the wavelength shifting fibers in the sigma groove are painted with reflective paint to to maximize the light detection. It has been observed that three turns gives twice as much light compared to a single turn of the fiber [14]. Panel (a) of Fig. 2 shows the route of a wavelength shifting fiber of a tile in a super-sector. Finally the edge of the scintillator wedge is polished to increase the reflectivity, wrapped with one layer of Tyvek and two layers of black paper. Silicon photomultipliers (SiPM) are used for light detection and signal amplification. To reduce radiation damage to the SiPMs by neutrons in the high radiation forward region, $5.5 \mathrm{~m}$ long clear fiber (Kuraray $1.15 \mathrm{~mm}$ in diameter) is used to carry the light from the EPD tiles to the SiPMs. To reduce the light loss during transmission from the wavelength shifting fiber to the clear fiber, a slightly larger clear fiber is used. A clear fiber bundle ends in a 31 channel connector at the super-sector, and at the other end the fibers are grouped in two bundles of 15 and 16 fibers. All the connectors and parts of the clear fiber bundles 
are custom made using 3D-printing technology. Black polylactic acid (PLA) plastic is used as the material for 3D printing. The SiPMs are Hamamatsu S13360-1325PE MPPC devices, which have a pixel pitch of $25 \mu \mathrm{m}$, with an effective photosensitive area of $1.3 \times 1.3 \mathrm{~mm}$, and a total of 2668 pixels. A sixteen channel SiPM board was developed to connect the SiPMs to the far end of the clear fiber bundle. The SiPM board is attached to the clear fiber with a spacer ( $\sim 1 \mathrm{~mm}$ thickness) to prevent scratching of the SiPM surface. The SiPM board connects to a 16 channel FEE board, which is read by the existing STAR trigger and DAQ system [15].

\section{Achievements from Prototype Run}
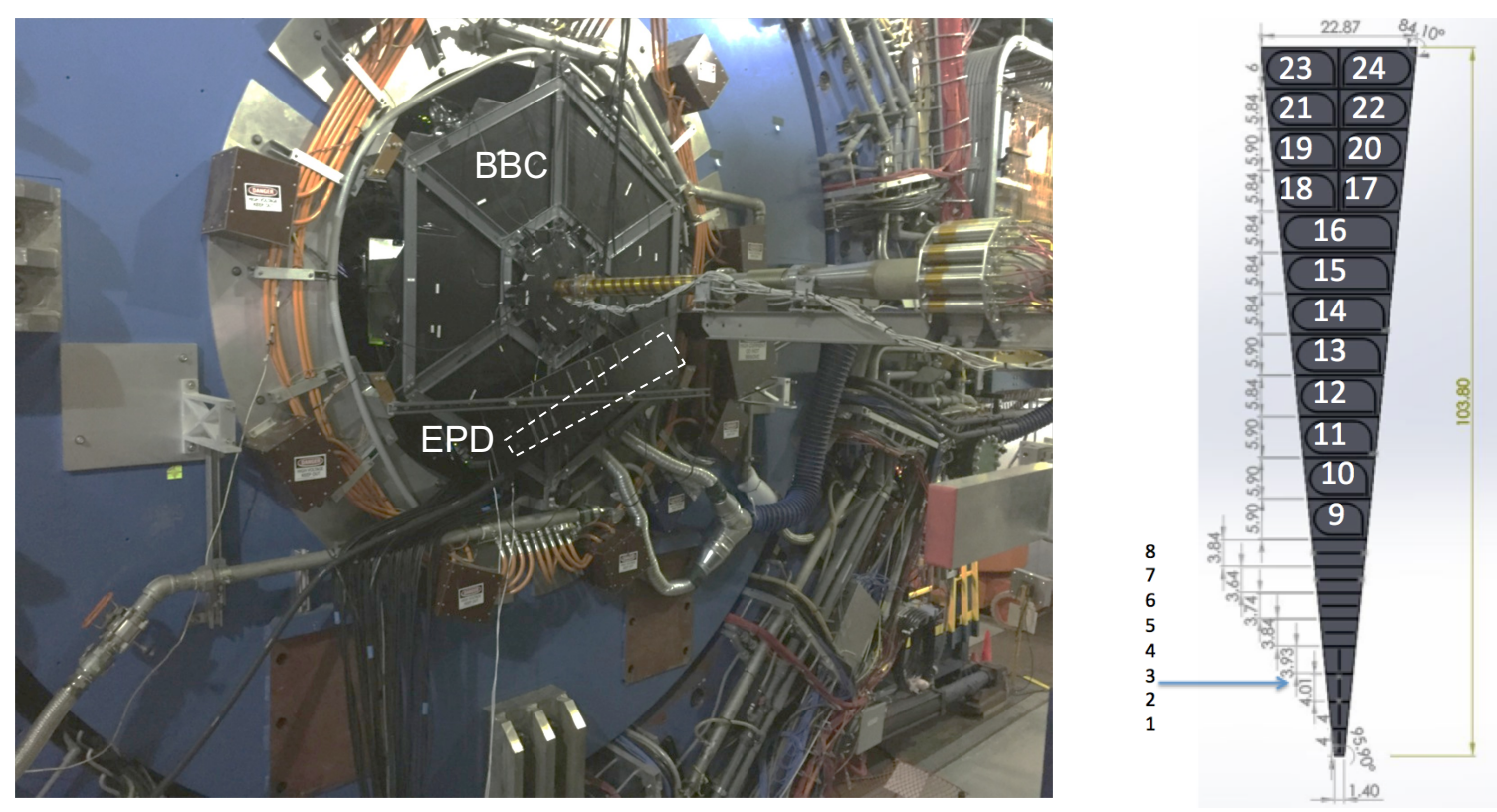

Figure 3: On the left is a photo of the EPD prototype installed in the east side of STAR during Run16. The white lines are drawn in order to guide the eye. On the right is a diagram of the prototype EPD tiles with the tile numbers labeled.

During Run16, a prototype of a 24 tile sector was installed on the east side of the STAR experiment. The location of the sector in STAR and arrangement of the 24 tiles in the sector is shown in Fig. 3. This prototype tile geometry was different from the final design and were mainly used for the purpose of research and development of tile geometry, wavelength shifting fiber groove geometry, fiber-fiber and fiber SiPM coupling techniques. The prototype recorded data during $200 \mathrm{GeV}$ $\mathrm{Au}+\mathrm{Au}$ collisions. Panel (a) of Fig. 4 shows an ADC (Analog-to-Digital Conversion) distribution from tile 14, which is a tile closer to the beam pipe. The ADC distribution is a convolution of many particle hits, pedestal signal, electronic noise and so on. To understand the number of particles that hit a tile, a fit consisting of the convolution of a Landau distribution, Poisson distribution, Gaussian distribution and exponential distribution is done. Using the convoluted fitting function we were able to identify multiple hit events from the ADC distribution.

Panel (b) of Fig. 4 shows the timing capabilities of the EPD. In the plot the vertical axis TAC (Time-to-Amplitude converted) ADC value measures the timing of particle hits in EPD tiles. The 
plot reveals the band structure of the RHIC RF system and each band is expected to be separated by $5 \mathrm{~ns}[16]$.

Wavelength shifting fibers used in the EPD have a minimum bending radius of $2.5 \mathrm{~cm}$. To avoid over-bending the fibers, the inner tiles have a straight line fiber geometry. However the larger tiles were able to have sigma shaped grooves. The prototype and lab testing showed that the sigma groove geometry was capable of single photon detection, and better signal strength and tile coverage. Therefore we have adopted the sigma shaped grooves for embedding Wavelength shifting fibers in the scintillator tiles.
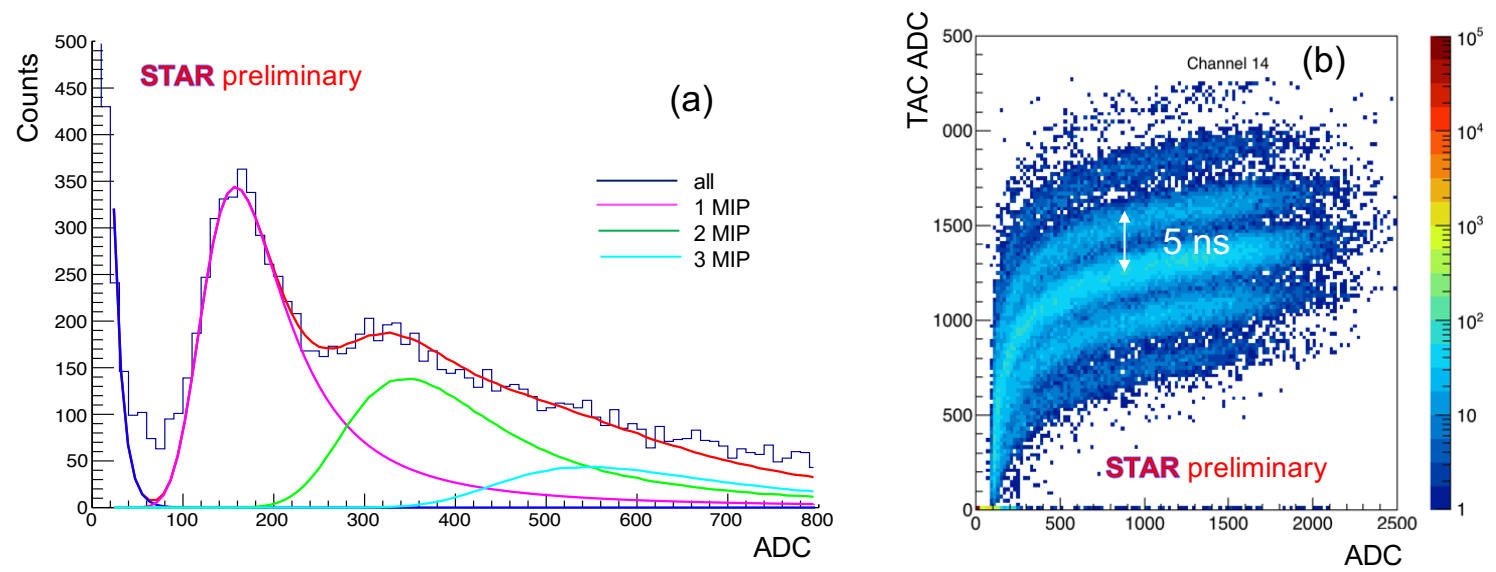

Figure 4: On the left is an ADC spectrum of tile 14 and the fit extracting (see text) 1,2,3 MIPs from Run16 $\mathrm{Au}+\mathrm{Au} 200 \mathrm{GeV}$ data. On the right ADC vs. TAC ADC values for the same tile as shown. TAC ADC channels have a timing resolution of $20 \mathrm{ps} /$ count.

\section{Results from Engineering Run for detector commissioning}

During Run 17, $1 / 8^{\text {th }}$ (quarter wheel) of the EPD installed on the east side of STAR (see Fig. 5). A quarter wheel consist of three super-sectors and 93 channels. The supers-sectors are placed on a custom made strong-back. The strong-back places the super-sectors very close to the beam pipe which leaves about $1 \mathrm{~cm}$ space between inner tile and the beam pipe. The detector was commissioned during p+p $500 \mathrm{GeV}$ collisions. The bias voltage of the SiPMs were adjusted to roughly equalize the MIPs of the tiles. The detector was fully functional during the subsequent $\mathrm{Au}+\mathrm{Au} \sqrt{s_{N N}}=54.4 \mathrm{GeV}$ collisions in Run17 and was able to take useful data. Clear MIP peaks were seen in all the tiles, and uniform response was seen from all the tiles across the detector. Panel (a) and (b) of Fig. 6 show two such ADC distributions from two tiles, which are at the extreme pseudorapidity of the detector. The ADC distribution is also fitted as described above to identify events with multiple hits, about $50 \%$ of the events have 1-MIP events and about $1 \%$ of the events have 5-MIP events. The fit also indicates about 45 photons per MIP. The Run 17 operation shows a SiPM dark-current of the order of $1 \mu A$ and no degradation of the MIP resolution. We expect much less radiation damage in $\mathrm{Au}+\mathrm{Au}$ collisions. 

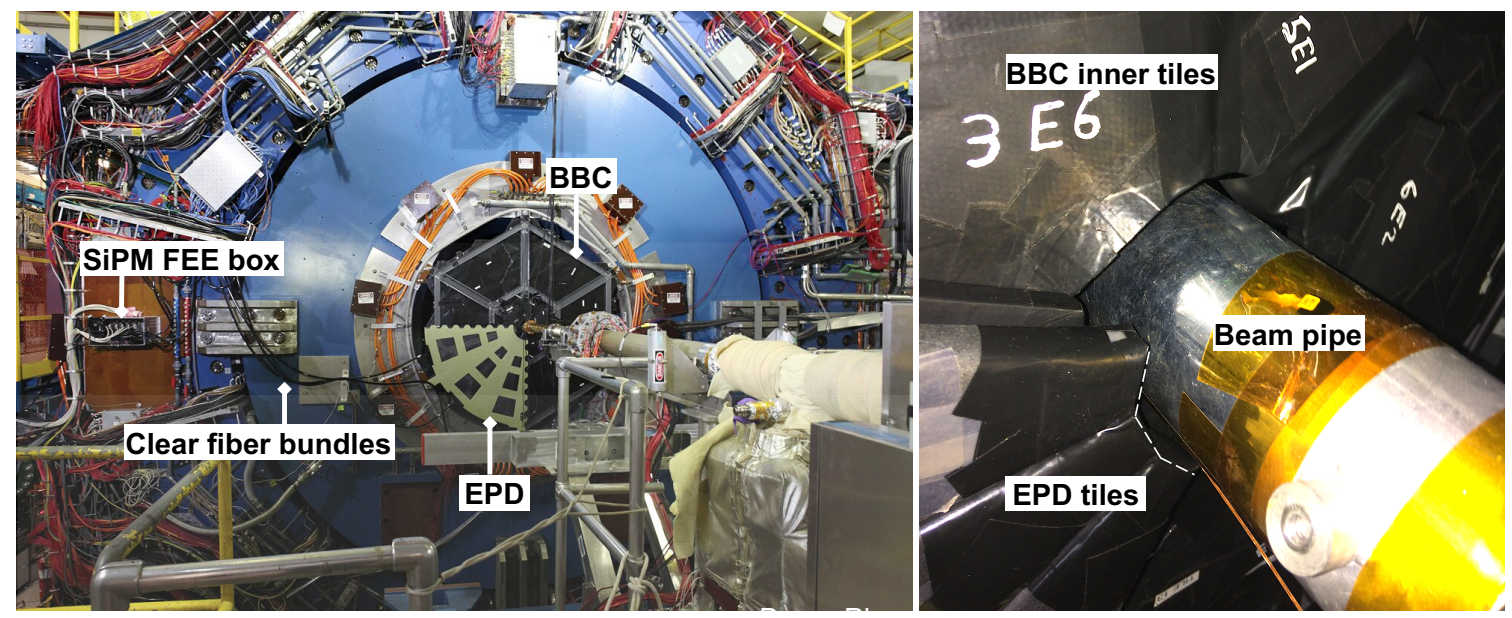

Figure 5: On the left EPD quarter wheel installed on the east side of STAR for Engineering Run. On the right closer look of the inner tiles of BBC, EPD and beam pipe. The white lines are drawn in order to guide the eye to show how the inner tiles extend closer to beam pipe.
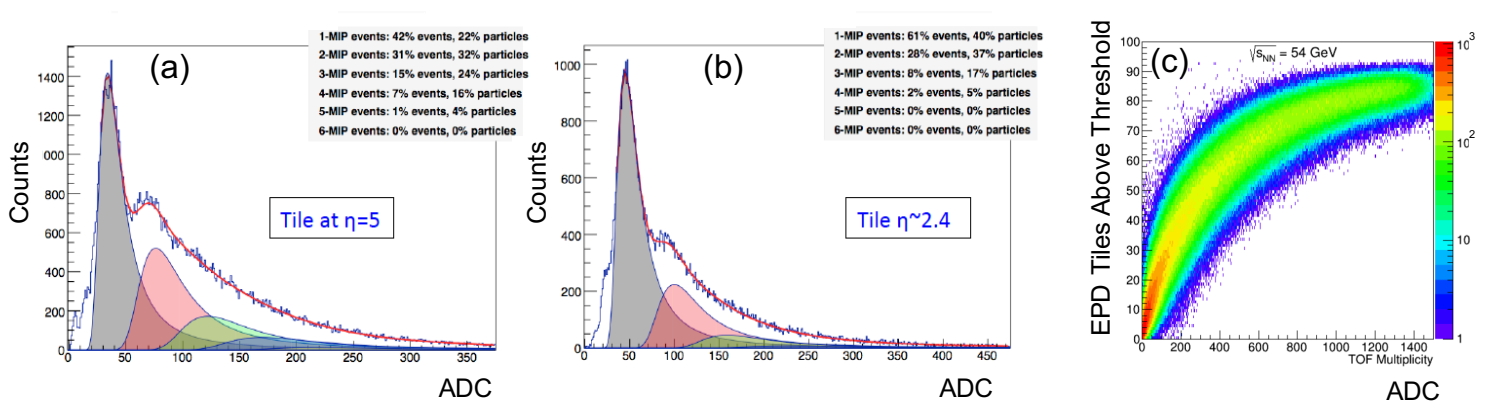

Figure 6: Panel (a) presents ADC distribution of a tile at higher pseudorapidity (closer to beam pipe). Plot also shows the events in the distribution identified for multiple MIPs from the fit. Similarly panel (b) presents ADC distribution for a tile at low pseudorapidity. Panel (c) of the figure shows correlation between the multiplicity of particles detected by mid-rapidity detector TOF [17] and number of EPD tiles above threshold to make a hit.

\section{Conclusions and Outlook}

The Event Plane Detector fulfills critical requirements for the phase two of the STAR's beam energy scan program. The EPD opens up the phase-space and provides significant azimuthal and radial segmentation in the forward rapidity reagion. This improves the event plane resolution and allows measurements of centrality at forward rapidity. The detector showed excellent performance during the engineering run and data with $\mathrm{Au}+\mathrm{Au} 54.4 \mathrm{GeV}$ collisions were recorded. Phase two of the BES program needs efficient background reduction for high-luminosity RHIC collisions. The EPD design exceeds minimum radial segmentation needed for an effective minimum-bias trigger. During Run17 EPD met our goal of integrating the EPD into the STAR trigger framework and utilizing it as a trigger detector.

The detector is on schedule for completion. The full detector will be installed before beginning 
of the RHIC Run18, and functional for Isobar and BES II collisions. The event plane detector together with other STAR detector upgrades and low energy electron cooling provides good control of statistical fluctuations and systematics for BES II.

\section{References}

[1] I. Arsene et al. (BRAHMS Collaboration), Nucl. Phys. A 757, 1 (2005); B. B. Back et al. (PHOBOS Collaboration), Nucl. Phys. A 757, 28 (2005); J. Adams et al. (STAR Collaboration), Nucl. Phys. A 757, 102 (2005); K. Adcox et al. (PHENIX Collaboration), Nucl. Phys. A 757, 184 (2005); M. M. Aggarwal et al. (STAR collaboration), arXiv:1007.2613; STAR Note SN0493 (2009); S. A. Bass et al., Hot \& Dense QCD Matter, Whitepaper submitted to the 2012 Nuclear Science Advisory Committee.

[2] STAR collaboration, BES Phase-II Whitepaper, STAR Note SN0598 (2014).

[3] L. Adamczyk et al. (STAR Collaboration), Phys. Rev. Lett. 112, 032302 (2014).

[4] Xiaofeng Luo (STAR Collaboration), PoS(CPOD2014)019 (2015).

[5] L. Adamczyk et al. (STAR collaboration), Phys. Rev. Lett. 112, 162301 (2014).

[6] L. Adamczyk et al. (STAR collaboration), arXiv:1708.07132 [hep-ex].

[7] L. Adamczyk et al. (STAR collaboration), Nature 548, 62 (2017)

[8] C. A. Whitten (STAR Collaboration), AIP Conf. Proc. 980 (2008) 390.

[9] D. H. Rischke et al., Heavy Ion Phys. 1, 309 (1995).

[10] H. Stöcker, Nucl. Phys. A 750, 121 (2005).

[11] B. Alver et al., (PHOBOS Collaboration), Phys. Rev. C 83, 024913 (2011).

[12] https://drupal.star.bnl.gov/STAR/files/Construction_Proposal_0.pdf

[13] M. Olsson et al., University of Rochester preprint UR-1370 (1994).

[14] S. Filippov et al., LHCb 2000-31 CALO (2000).

[15] F.S. Bieser et al., Nucl. Instr. Meth. A 499, 766 (2003); H.J. Crawford et al., http://hena.lbl.gov/FMS/cap-equip-qt.pdf

[16] T. Satogata, https://www.bnl.gov/isd/documents/43565.pdf

[17] W.J. Llope (STAR Collaboration), Nucl. Inst. and Methods A 661 (2012) S110 